Article

\title{
Increasing Incidence of Mucormycosis in Spanish inpatients from 1997-2018
}

\author{
Raúl Parra Fariñas ${ }^{1}, \mathrm{MD}$, Montserrat Alonso-Sardón², MD, PhD, Virginia Velasco-Tirado ${ }^{3}, \mathrm{MD}$, PhD, Inmaculada \\ Galindo Pérez ${ }^{4}, \mathrm{MD}$, Cristina Carbonell ${ }^{5}$, MD, Elisa Álvarez Artero ${ }^{6}$, Ángela Romero-Alegría ${ }^{7}, \mathrm{MD}$, PhD, Javier \\ Pardo-Lledías $^{8 *}$, MD, PhD, Moncef Belhassen-García ${ }^{9 *}$, MD, PhD.
}

* Both authors have contributed equally as senior authors of this manuscript.

1 Servicio de Medicina Interna, Hospital Marqués de Valdecilla, Universidad de Cantabria, Spain. E-mail: raul.parra@scsalud.es

2 Area of Preventive Medicine and Public Health, University of Salamanca, Instituto de Investigación Biomédica de Salamanca (IBSAL), Centro de Investigación de Enfermedades Tropicales de la Universidad de Salamanca (CIETUS), Salamanca, Spain. E-mail: sardonm@usal.es

3 Servicio de Dermatología, Complejo Asistencial Universitario de Salamanca (CAUSA), CIETUS, IBSAL, Salamanca, Spain. E-mail: virvela@yahoo.es

4 Centro de Atención Primaria, Santoña, Cantabria, Spain. E-mail: igalindop@hotmail.com

5 Servicio de Medicina Interna, CAUSA, IBSAL, CIETUS, Salamanca, Spain. E-mail: crstncarbonell@gmail.com

6 Servicio de Medicina Interna. Hospital Rio Carrión. Complejo Asistencial de Palencia (CAUPA). Avenida Donantes de Sangre S/N, 34005, Palencia, España. E-mail: e.alvarez.artero@gmail.com

7 Servicio de Medicina Interna, CAUSA, IBSAL, CIETUS, Salamanca, Spain. E-mail: aralegria@yahoo.es

8 Servicio de Medicina Interna, Hospital Marqués de Valdecilla, Universidad de Cantabria, Instituto de Investigación Valdecilla (IDIVAL), Spain. E-mail: javier.pardo@scsalud.es

9 Internal Medicine, Infectious Diseases Section, CAUSA, IBSAL, CIETUS, University of Salamanca, Paseo San Vicente 58-182, 37007, Salamanca, Spain. E-mail: mbelhassen@hotmail.com

* Correspondence: Moncef, Belhassen García.E-mail: mbelhassen@hotmail.com e-mail@e-mail.com; Tel.: +34 923291306

\begin{abstract}
Background: Mucormycosis is a worldwide angio-invasive fungal infection that is associated with high morbidity and mortality. A few European studies have focused on the epidemiology. Methodology: A retrospective longitudinal descriptive study was performed with inpatients diagnosed with mucormycosis (ICD-9-CM, code 117.7, cases 1997-2015; and ICD-10, code B46, cases 2016-2018; along with length of hospital stay) in Spanish public hospitals between 1 January 1997 and 31 December 2018. Data were obtained from the Minimum Basic Data Set (CMBD in Spanish). Principal findings: A total of 962 patients were recorded; 665 were men. The mean age $( \pm$ SD) was $55 \pm 18.8$ years. The annual incidence rate increased from 0.74 to 1.24 cases per million person-years. The lethality rate was $31.3 \%$. Renal failure $(41.6 \%)$ and hematological malignancy (36.3\%) were the main factors involved. Conclusions: Mucormycosis is a rare infectious disease in Spain, but it has had a significantly increased incidence in the last two decades. Being an adult male and having diabetes, neoplasm or renal failure are the main factors associated. High mortality is usually associated mainly with hematological malignancy and renal failure. CMBD studies could be an efficient tool for assessing changes in the epidemiology of mucormycosis.
\end{abstract}

Keywords: Mucormycosis; Zygomycosis; Epidemiology; Incidence; Clinical; Spain.

\section{Introduction}

Mucormycosis is an angio-invasive infection caused by a group of fungi belonging to the order of Mucorales. Rhizopus is considered the most frequent cause of this fungal infection in humans, followed by Mucor and Rhizomucor; Cunninghamella, Lichthemia, Saksenaea and Apophysomyces are genera that are less commonly implicated in human infection [1]. Mucorales are distributed worldwide and are found ubiquitously in the environment; 
they are thermotolerant fungi found in soil and decaying matter and rarely cause disease because of their low virulence. The most commonly encountered clinical form is rhinocerebral mucormycosis, which is acquired by the inhalation of spores. In healthy individuals, cilia transport these spores to the pharynx, and they are cleared through the gastrointestinal tract [2]. In immunocompromised individuals, infection usually begins in the nasal turbinates or the alveoli, and the resultant mortality, even with pharmacological and/or surgical treatment, is high unless the immune system status can be restored. Risk factors include diabetes mellitus, particularly with ketoacidosis, malnutrition, malignancies (lymphomas and leukemias), organ transplant, trauma, burns, and immunosuppressive therapy [3-8]. Patients on dialysis who receive treatment with the iron chelator deferoxamine are also more susceptible to mucormycosis [9]. Mucormycosis is less common in patients with AIDS because $\mathrm{T}$ cell-mediated immunity is not considered an important factor for triggering the infection [10]. The real incidence of mucormycosis is difficult to estimate since it is not a reportable disease and the risk varies widely in different populations and geographic regions [11]. A change in the incidence of mucormycosis is expected to be associated with an improved lifespan in cancer and transplant patients, as well as expanding indications for immunosuppressive medications for various autoimmune diseases. The aim of this study was to estimate the incidence of mucormycosis in Spain and to describe the epidemiological and clinical characteristics of patients hospitalized in the National Health System (SNS) between 1997 and 2018.

\section{Materials and Methods}

\subsection{Study design and population}

This is a retrospective longitudinal descriptive study of hospitalized patients diagnosed with zygomycosis/mucormycosis in public hospitals of the Spanish National Health System (NHS) between January 1, 1997, and December 31, 2018. Inclusion criteria: All patients admitted to public hospitals of the NHS between 1997 and 2018 with a principal and/or secondary diagnosis of zygomycosis/mucormycosis according to the International Classification of Disease (ICD) codes 9th revision-Clinical Modification, ICD-9-CM Diagnosis Code 117.7 Zygomycosis [Phycomycosis or Mucormycosis], cases 1997-2015; and 10th revision-Clinical Modification, CD-10-CM Diagnosis Code B46 Zygomycosis [B46.0 Pulmonary mucormycosis; B46.1 Rhinocerebral mucormycosis; B46.2 Gastrointestinal mucormycosis; B46.3 Cutaneous mucormycosis; B46.4 Disseminated mucormycosis; B46.5 Mucormycosis, unspecified; B46.8 Other zygomycoses; B46.9 Zygomycosis, unspecified], cases 2016-2018. Exclusion criteria: Patients with missing data were excluded from the study.

\subsection{Data collection}

Data were obtained from the Specialized Health Care Registry (RAE-CMBD in Spanish), which includes data on hospitalization, outpatient surgery, day hospitalization, and home and emergency hospitalization. RAE-CMBD is the main Health Information System used to obtain statistical data and basic health indicators on morbidity and the care process of patients treated in Spanish hospitals. It contains information on general data (age, sex, and place of residence), morbidity data and procedures performed (principal diagnosis or secondary diagnosis), variables related to the episode of hospitalization, circumstance of admission (urgent or planned), patient discharge (discharge to home address, transfer to another hospital or death), stay and average cost.

\subsection{Data analysis}

The incidence rate was calculated by dividing the number of new cases of zygomycosis/mucormycosis (numerator) per year/period by the population at risk (denominator) over a period of time (person-years) multiplied by 1,000,000 and expressed as "cases per million person-years". As it is not possible to accurately measure disease-free periods, the 
total figure of person-time at risk can be estimated approximately and satisfactorily when the size of the population is stable, multiplying the average population size studied by the duration of the observation period. Thus, the population at risk was obtained from annual data published by the National Institute of Statistics (INE, http://www.ine.es/;population of Spain in 1997: 39,323,320 inhabitants; population of Spain in 2018: 46,722,980 inhabitants). The 95\% confidence interval (95\% CI) for the incidence rate was calculated for a better clinical application of the results. Incidence rates were computed by autonomous community and year to assess temporal and geographical patterns. The results in terms of mean rates by autonomous community were plotted in maps for the whole study period. The lethality rate was calculated by dividing the number of principal diagnosis deaths (numerator) by the number of sick patients with a principal diagnosis of a specific disease (denominator) (x100). Pearson's correlation coefficient $(\mathrm{rP})$ was calculated to assess the correlation between linear variables, incidence rate and environmental parameters. The weather parameters were obtained from the State Agency of Meteorology (AEMET, in Spanish, http://www.aemet.es/). The results are expressed as absolute values (n), proportions $(\mathrm{n} / \mathrm{N})$ and percentages $(\%)$ for categorical variables and as the mean, standard deviation (SD), median, interquartile range (IQR) (Q3-Q1), and range (minimum value, maximum value) for continuous variables. The strength of the association between categorical variables was measured using Pearson's $\chi 2$ contrast statistic and the odds ratio (OR) estimate. Continuous variables were compared with Student's t-test or the Mann-Whitney test for two groups, depending on their normal or non-normal distribution. ANOVA (F-test) was used to statistically assess the equality of means between groups. The level of statistical significance was $p<0.05$. Data analysis was performed using SPSS 26 (Statistical Package for the Social Sciences).

\subsection{Ethics Statement}

This study is based on medical data of patients collected in the CMBD. These data are the responsibility of the Ministry of Social Services of Health and Equality (Ministerio de Servicios Sociales, Sanidad e Igualdad, MSSSI) that maintain custody and organize them. All patient data provided by the CMBD are anonymized and deidentified by the MSSSI before they are provided to the applicants. According to this confidentiality commitment signed with the MSSSI, researchers cannot provide the data to other researchers, so other researchers must request the data directly from the MSSSI. The protocol and ethics statement of this study were approved by the Clinical Research Ethics Committee of the Complejo Asistencial Universitario de Salamanca (CAUSA). Because the data were obtained from an epidemiological database, written consent was not obtained. All data were analyzed anonymized.

\section{Results}

A total of 962 cases with ICD-9-CM Diagnosis Code 117.7 and ICD-10-CM Diagnosis Code B46 were registered in Spain during the 22-year study period, 1997-2018. The period incidence rate was 0.98 (95\% CI, 0.92-1.04) cases per million person-years. The annual incidence rate increased from 0.74 (95\% CI, 0.45-1.00) cases per million person-years (29 cases) in 1997 to 1.24 (95\% CI, 0.92-1.56) cases per million person-years (58 cases) in 2018 (see Figure 1). The annual incidence rate was 1.46 (95\% CI, 1.11-1.81) cases per million person-years (68 cases) in 2016 and the annual incidence rate was 0.49 (95\% CI, $0.28-0.69$ ) cases per million person-years (21 cases) in 2004. The incidence rate increased significantly in the second half of the study: 1.19 (95\% CI, 1.10-1.29) vs. 0.75 (95\% CI, 0.67-0.83); $(\mathrm{p}<0.001)$.

A higher number of cases $(277 ; 28.8 \%)$ occurred in the summer months: August (96; $10 \%)$ and September $(101 ; 10,5 \%)$ were the months with the highest incidence of cases (see Figure 2). No seasonal variability was observed over the 22-year study period $(p=0.100)$. We assessed whether environmental parameters, such as temperature and 
precipitation, could condition variations between Spanish regions and over the study period (1997-2007 vs. 2008-2018). There was no statistically significant linear association between temperature, rainfall or autonomous community incidence rate $(\mathrm{rP}=0.021$; $\mathrm{p}=0.933$ and $\mathrm{rP}=0.156 ; \mathrm{p}=0.524$, respectively) or temperature, rainfall and time period incidence rate $(\mathrm{rP}=0.324 ; \mathrm{p}=0.142$ and $\mathrm{rP}=-0.143 ; \mathrm{p}=0.525$, respectively) (see Figure 3 ).

Figure 4 shows the geographical distribution (by autonomous communities) of mucormycosis cases in Spain. The highest incidence rates corresponded to the western and northern regions of the Iberian Peninsula: Extremadura, 1.67 (95\% CI, 1.54-2.19) cases per million person-years; Castilla y León, 1.29 (95\% CI, 0.99-1.59) cases per million person-years; and Cantabria, 1.52 (95\% CI, 0.84-21.21) cases per million person-years.

The characteristics of the patients included in the study and the predisposing factors are shown in Table 1 . The number of men $(665 ; 69.1 \%)$ was double that of women (297; $30.9 \%$ ), as shown by the incidence rate in men, 1.38 (95\% CI, 1.28-1.49) cases per million person-years, vs. women, 0.60 (95\% CI, 0.53-0.67) cases per million person-years. The mean $( \pm S D)$ age was 55 years $( \pm 18.8)$ [median $(I Q R), 58$ years $(69-44)$ ]. The percentage of cases in the pediatric population (under 14 years old) was low (3.1\%). Hospitalizations with ICD-9-CM code 117.7 or ICD-10 code B46 as the principal diagnosis code were 348 $(36.2 \%)$ vs. $614(63.8 \%)$ cases with one of these as the secondary diagnosis code. A total of 215 cases $(22.3 \%)$ were treated by the hematology service, and $148(15.4 \%)$ were treated by the internal medicine service. The average $( \pm \mathrm{SD})$ hospital stay was 32 days $( \pm 34.1)$ [median (IQR), 22 (43-8)]; it was higher in patients with secondary diagnosis [mean \pm SD, $34.9 \pm 37.7$ vs. $26.1 \pm 25.8 ; \mathrm{p}<0.001]$. Diabetes mellitus $(33.6 \%)$ and hematologic malignancy $(32.6 \%)$ were the main disorders associated with mucormycosis. Diabetes mellitus involvement was similar in the first and second half of the study $(32.3 \%$ vs. 34.3\%; $\mathrm{p}=0.522)$. Renal failure $(14.4 \%$ vs. $24.7 \%$; $<0.001)$ and nonhematological malignancy $(6.9 \%$ vs. $13.7 \%$; $=0.001)$ increased significantly in the second half of the study period, while HIV decreased (5.2\% vs. $1.8 \%$; $=0.003)$.

The cohort lethality rate was $31.3 \%$. Risk factors associated with mortality are shown in Figure 5. Renal failure $(41.6 \%, \mathrm{p}<0.001)$ and hematological malignancy $(36.3 \%, \mathrm{p}=$ 0.019) were associated with excess mortality. In contrast, the absence of risk factors was associated with a lower fatality rate $(22.3 \%, \mathrm{p}=0.006)$. The lethality rate associated with diabetes mellitus was similar in the first and second halves of the study $(33.3 \%$ vs. $32.1 \%$; $\mathrm{p}=0.830)$. The lethality rate associated with renal failure $(14.8 \%$ vs. $35.2 \%$; $\mathrm{p}<0.001)$ increased significantly in the second half of the study period.

We estimated the global cost of this cohort of patients with principal or secondary diagnosis of mucormycosis in Spain (from 1997 to 2018) to be approximately $€ 13,054,795.20$ [average $( \pm S D)$ cost per patient, $€ 13,570.47( \pm 17,257.58)$; median cost, $€ 6,885.13]$. It is important to note that the cost was higher in the patients who had a surgical procedure [mean $\pm S D, € 23,468.08 \pm 23,542.14]$ vs. a medical procedure [mean $\pm S D$, $€ 8,490.10 \pm 8,712.31 ; \mathrm{p}<0.001]$. The average cost was also higher in patients with a secondary diagnosis [mean $\pm S D, € 15,373.87 \pm 19,026.65$ vs. $€ 10,388.61 \pm 13,014.56 ; p<0.001]$.

\section{Discussion}

Mucormycosis is the third most common invasive fungal infection, following aspergillosis and candidiasis [12], and it has a significant degree of morbidity and mortality. During the study period, 962 patients admitted for mucormycosis were registered, with a period incidence rate of 0.98 cases per million person-years. These data show that mucormycosis is an extremely rare infection in Spain, with incidences up to thirty times lower than those observed for Aspergillus, as previously shown [13]. However, the true incidence of mucormycosis may be higher, as many cases remain undiagnosed due to the difficulty in collecting samples from deep tissues and the low sensitivity of diagnostic tests. Nevertheless, during the study period, we detected a significant increase in the number of cases; in the second decade of the study, we found that the incidence of mucormycosis in Spain increased by $67 \%$ compared to the first part of the study. These data 
show an increasing number of cases in Spain that exceeds the estimates in other areas, such as Asia (31\%) and North or South America (28\%), Africa (3\%), Australia and New Zealand (3\%) [14]. The rise is also very high in India and China among patients with uncontrolled diabetes mellitus [15]. In Europe, a few studies have focused on the incidence of mucormycosis, but it is not feasible to obtain exact incidence rates [16]. In a large national multicenter 10-year study, Bitar et al. [17] showed an increasing incidence from 0.7 per million in 1997 to 1.2 per million in 2006. Another study conducted in metropolitan France from 2001-2010 showed that the incidence of mucormycosis increased by $7.3 \%$ per year [18]. In addition, a study conducted over a 9-year time period in Belgium found that the annual incidence rate increased from 0.0019 cases/10.000 patient-days in 2000 to 0.148 cases/10.000 patient-days in 2009[19]. Finally, in the largest review of mucormycosis cases in the English language literature, Roden et al. concluded that there was a substantial increase in the reporting during the study period [1]. It is interesting that although very different methods were used to analyze the epidemiology, multiple studies have reported an increase in the incidence of mucormycosis as a result of the expansion of the population at risk and the use of prophylactic voriconzale [19], as well as the optimization of microbiology techniques for mucor isolation [3]. It is remarkable that the epidemiology of mucormycosis can vary with geographic region [11]. In our study, we investigated whether differences in climate could explain differences in the number of cases between different areas of our country, as suggested was the case for aspergillosis in a previous paper [13]. Although we detected a higher number of cases in the month of August, we did not find that rainfall, temperature or other factors were involved in differences associated with seasonality or differences in the incidence of this disease between different areas. Our data disagree with data shown by Rahal et al. [20], in which more than $60 \%$ of cases of mucormycosis occurred between May and August. The use of air conditioners and shared closed environments were postulated as possible explanations for this phenomenon [20]. In our study, the number of cases in men $(69.1 \%)$ was double that of women (30.9\%), which is in line with the results described in other works $[14,16,21]$. Another key epidemiological feature observed in our study was that the subjects were the same age as those in other works. Previous data showed that the median age of patients with mucormycosis varied from 50 to 54 years, which was similar to the mean age of 55 observed in our cohort [14,19,21]. We highlight in our work that more than $1 / 3$ of the patients are over 65 years of age and only $3 \%$ are pediatric ( $<14$ years). The main underlying diseases and conditions described in the literature vary between studies [16] and include i) hematological malignancies, including hematopoietic stem cell transplantation; ii) other malignancies, solid organ transplantation and other immune deficiencies, such as HIV/AIDS, liver cirrhosis, renal insufficiency, and high-dose steroids; iii) diabetes mellitus; and iv) trauma, including secondary to an accident, major surgery and burns. Among patients with malignancies, hematologic malignancies are much more frequently associated with mucormycosis than solid tumors [7]. We emphasize that there are several differences in the prevalence of risk factors associated with mucormycosis between different areas worldwide. For example, in Europe and the United States, hematological malignancy (HM)[15] is the main factor involved (from $38 \%$ to $62 \%$ of the cases), whereas diabetes mellitus is the main factor in Asian countries [14,21,22]. These data were similar to our results, with either diabetes or HM involved in more than one-third of all patients diagnosed with mucormycosis. In our work, we studied changes in the main factors associated with mucormycosis during the study period. We did not detect differences in the frequency of diabetes in the study population between the two parts of study, i.e., 1997-2007 vs. 2008-2018. These data are consistent with other European countries, such as France, in which the rate of mucormycosis cases in patients with diabetes has not decreased in recent years $[17,22]$. However, a study performed in other areas of Western countries (EEUU) found an increase in cases in patients with uncontrolled diabetes [22]. It is possible that the increase in the prevalence of diabetes in Spain in the last two decades could be counteracting the effects of better metabolic control ex- 
pected with the arrival of new, more effective treatments in the management of this disease. Conversely, we found that other classic risk factors, such as renal failure and nonhematological malignancy, increased in the second part of the study, probably due to higher life expectancy. In addition, in recent years, a considerable number of cases have been documented following trauma in apparently healthy individuals, and health-care-associated mucormycosis is being increasingly documented [23]. In this study, unfortunately, we did not have the locations or species involved in the mucormycosis cases to analyze and compare to other reports [3]. The fact that the majority of patients were diagnosed by histopathology and later in the disease course (i.e., postmortem diagnosis in $16.4 \%$ ) underscores the eminent need to validate rapid serological and molecular diagnostic tests for mucormycosis. Mortality varied in the different studies and according to different underlying conditions and location of infection, with much higher rates among patients with hematologic malignancies, disseminated infection and antifungal therapy alone compared to those receiving a combination of antifungal treatment and surgery [16,24]. In our work, the overall lethality rate was $31.3 \%$, which was high but within the range of other studies showing global mortality between 36-65\% $[19,21,24-26]$. Notably, there was low mortality among HIV patients $(20.7 \%)$ in our study compared to other studies on HIV that reported a mortality of 55\% [3]. The differences in these data could be due to differences in treatment strategies. Unfortunately, due to the methodology of our study, we do not know the treatment selected or the location of mucormycosis, which could explain these differences [25]. We also assessed the economic cost of mucormycosis in our country after not finding any literature about it. Health expenditure varies according to the different economic conditions of each country and the health care systems. Our data show that the total cost of mucormycosis per person is significant. The mean cost in Spain was higher than the total cost of the most prevalent inpatient pathology (e.g., pneumonia, heart failure, ischemic stroke) and similar to the cost of sepsis (http://www.msssi.gob.es/). It should be noted that the estimated cost is only the cost due to hospitalization, and other costs are not accounted for, so the final sum would be higher.

Strengths and limitations

The CMBD is a database that continually collects information on hospital admissions for most of our hospitals, so the representativeness of the sample is very appropriate. ICD code-based evaluations have limitations; such limitations are mainly related to difficulties encountered with the diagnosis of mucormycosis in clinical practice. Conventional diagnostic tools lack sensitivity. Moreover, the distinction between colonization (a positive culture does not always mean infection, as these organisms are common in the environment) [16] and infection is problematic in the absence of invasive procedures or autopsy data. Thus, epidemiologic studies on mucormycosis are often hampered by the limited number of documented cases. Initiatives, such as the Fungiscope, a Global Registry for Emerging Fungal Infections, have contributed significantly to the current knowledge of rare fungal infections, including mucormycosis. In Europe, the largest registry for mucormycosis is the one constructed by the earlier European Confederation of Medical Mycology (ECMM) and current ECMM/ISHAM Working Group on Zygomycosis (www.zygomyco.net). The existence of a single European registry allows focus on the clinical cases and mucoralean species encountered in Europe and ensures that cases are not submitted in duplicate [16]. Despite these limitations, the CMBD is a valuable tool that complements active records for developing research and adds value to scientific knowledge and clinical care in the field of medicine.

We have demonstrated that mucormycosis is a rare infectious disease in Spain, but it has had a significantly increased incidence in the last two decades. Being an adult male and having diabetes, neoplasm or renal failure are the main factors associated with this infection. Renal failure and nonhematological malignancies may be the main factors involved in this increase. Seasonality and weather conditions are not involved in the epi- 
demiology of this infection in our area. High mortality is usually associated mainly with hematological malignancy and renal failure. Finally, CMBD studies could be an efficient tool for assessing changes in the epidemiology of mucormycosis.

Author Contributions: "Conceptualization, J.P-Ll. and M.B-G.; methodology, M.A-S.; formal analysis, M.A-S., J.P-Ll and M.B-G.; investigation, R.P-F., V.V-T., I.G-P, C.C., E.A-A and A.R-A.; data curation, R.P-F., V.V-T., I.G-P., C.C. , E.A-A and A.R-A.; writing-original draft preparation, M.A-S., J.P-Ll. and M.B-G.; writing-review and editing, R.P-F., M.A-S., V.V-T., I.G-P., C.C., E.A-A, A.R-A., J-P-Ll and M.B-G.; supervision, J.P-Ll and M.B-G. All authors have read and agreed to the published version of the manuscript."

Funding: None declared.

Institutional Review Board Statement: This study is based on medical data of patients collected in the CMBD. These data are the responsibility of the Ministry of Social Services of Health and Equality (Ministerio de Servicios Sociales, Sanidad e Igualdad, MSSSI) that maintain custody and organize them. All patient data provided by the CMBD are anonymized and deidentified by the MSSSI before they are provided to the applicants. According to this confidentiality commitment signed with the MSSSI, researchers cannot provide the data to other researchers, so other researchers must request the data directly from the MSSSI.

Informed Consent Statement: The protocol and ethics statement of this study were approved by the Clinical Research Ethics Committee of the Complejo Asistencial Universitario de Salamanca (CAUSA). Because the data were obtained from an epidemiological database, written consent was not obtained. All data were analyzed anonymized.

Conflicts of Interest: The authors declare no conflict of interest.

\section{References}

1. Roden, M.M.; Zaoutis, T.E.; Buchanan, W.L.; Knudsen, T.A.; Sarkisova, T.A.; Schaufele, R.L.; Sein, M.; Sein, T.; Chiou, C.C.; Chu, J.H.; et al. Epidemiology and Outcome of Zygomycosis: A Review of 929 Reported Cases. Clin Infect Dis 2005, 41, 634-653, doi:10.1086/432579.

2. Ferguson, B.J. Mucormycosis of the Nose and Paranasal Sinuses. Otolaryng Clin N Am 2000, 33, 349-365, doi:10.1016/s0030-6665(00)80010-9.

3. Moreira, J.; Varon, A.; Galhardo, M.C.; Santos, F.; Lyra, M.; Castro, R.; Oliveira, R.; Lamas, C.C. The Burden of Mucormycosis in HIV-Infected Patients: A Systematic Review. J Infection 2016, 73, 181-188, doi:10.1016/j.jinf.2016.06.013.

4. Devauchelle, P.; Jeanne, M.; Fréalle, E. Mucormycosis in Burn Patients. J Fungi 2019, 5, 25, doi:10.3390/jof5010025.

5. Lanternier, F.; Sun, H.-Y.; Ribaud, P.; Singh, N.; Kontoyiannis, D.P.; Lortholary, O. Mucormycosis in Organ and Stem Cell Transplant Recipients. Clin Infect Dis 2012, 54, 1-8, doi:10.1093/cid/cis195.

6. Pappas, P.G.; Alexander, B.D.; Andes, D.R.; Hadley, S.; Kauffman, C.A.; Freifeld, A.; Anaissie, E.J.; Brumble, L.M.; Herwaldt, L.; Ito, J.; et al. Invasive Fungal Infections among Organ Transplant Recipients: Results of the Transplant-Associated Infection Surveillance Network (TRANSNET). Clin Infect Dis Official Publ Infect Dis Soc Am 2010, 50, 1101-11, doi:10.1086/651262.

7. Kontoyiannis, D.P.; Wessel, V.C.; Bodey, G.P.; Rolston, K.V.I. Zygomycosis in the 1990s in a Tertiary-Care Cancer Center. Clin Infect Dis 2000, 30, 851-856, doi:10.1086/313803.

8. Kontoyiannis, D.P.; Lionakis, M.S.; Lewis, R.E.; Chamilos, G.; Healy, M.; Perego, C.; Safdar, A.; Kantarjian, H.; Champlin, R.; Walsh, T.J.; et al. Zygomycosis in a Tertiary-Care Cancer Center in the Era of Aspergillus-Active Antifungal Therapy: A Case-Control Observational Study of 27 Recent Cases. J Infect Dis 2005, 191, 1350-1360, doi:10.1086/428780.

9. Boelaert, J.R.; Cutsem, J.V.; Locht, M. de; Schneider, Y.-J.; Crichton, R.R. Deferoxamine Augments Growth and Pathogenicity of Rhizopus, While Hydroxypyridinone Chelators Have No Effect. Kidney Int 1994, 45, 667-671, doi:10.1038/ki.1994.89.

10. Eucker, J.; Sezer, O.; Graf, B.; Possinger, K. Mucormycoses. Mycoses 2001, 44, 253-260, doi:10.1111/j.1439-0507.2001.00656.x.

11. Douglas, A.P.; Chen, S.C.-A.; Slavin, M.A. Emerging Infections Caused by Non-Aspergillus Filamentous Fungi. Clin Microbiol Infec 2016, 22, 670-680, doi:10.1016/j.cmi.2016.01.011.

12. Severo, C.B.; Guazzelli, L.S.; Severo, L.C. Capítulo 7 - Zigomicose. J Bras Pneumol 2010, 36, 134-141, doi:10.1590/s1806-37132010000100018.

13. González-Garcia, P.; Alonso-Sardón, M.; López-Bernus, A.; Carbonell, C.; Romero-Alegría, Á.; Muro, A.; Galindo-Pérez, I.; Muñoz-Bellido, J.L.; Pardo-Lledias, J.; Belhassen-García, M. Epidemiology of Aspergillosis in Hospitalised Spanish Patients-A 21-year Retrospective Study. Mycoses 2021, 64, 520-527, doi:10.1111/myc.13239. 
14. Jeong, W.; Keighley, C.; Wolfe, R.; Lee, W.L.; Slavin, M.A.; Kong, D.C.M.; Chen, S.C.-A. The Epidemiology and Clinical Manifestations of Mucormycosis: A Systematic Review and Meta-Analysis of Case Reports. Clin Microbiol Infec 2018, 25, 26-34, doi:10.1016/j.cmi.2018.07.011.

15. Prakash, H.; Chakrabarti, A. Global Epidemiology of Mucormycosis. J Fungi 2019, 5, 26, doi:10.3390/jof5010026.

16. Petrikkos, G.; Skiada, A.; Drogari-Apiranthitou, M. Epidemiology of Mucormycosis in Europe. Clin Microbiol Infec 2014, 20, 67-73, doi:10.1111/1469-0691.12563.

17. Bitar, D.; Cauteren, D.V.; Lanternier, F.; Dannaoui, E.; Che, D.; Dromer, F.; Desenclos, J.-C.; Lortholary, O. Increasing Incidence of Zygomycosis (Mucormycosis), France, 1997-2006. Emerg Infect Dis 2009, 15, 1395-1401, doi:10.3201/eid1509.090334.

18. Bitar, D.; Lortholary, O.; Strat, Y.L.; Nicolau, J.; Coignard, B.; Tattevin, P.; Che, D.; Dromer, F. Population-Based Analysis of Invasive Fungal Infections, France, 2001-2010. Emerg Infect Dis 2014, 20, 1163-1169, doi:10.3201/eid2007.140087.

19. Saegeman, V.; Maertens, J.; Meersseman, W.; Spriet, I.; Verbeken, E.; Lagrou, K. Increasing Incidence of Mucormycosis in University Hospital, Belgium. Emerg Infect Dis 2010, 16, 1456-1458, doi:10.3201/eid1609.100276.

20. E, M.R.; V, M.M.; N, J.V. Mucormicosis Rino-Orbito-Cerebral: Experiencia En Doce Años. Revista De Otorrinolaringología Y Cirugía De Cabeza Y Cuello 2008, 68, doi:10.4067/s0718-48162008000100005.

21. Skiada, A.; Pagano, L.; Groll, A.; Zimmerli, S.; Dupont, B.; Lagrou, K.; Lass-Florl, C.; Bouza, E.; Klimko, N.; Gaustad, P.; et al. Zygomycosis in Europe: Analysis of 230 Cases Accrued by the Registry of the European Confederation of Medical Mycology (ECMM) Working Group on Zygomycosis between 2005 and 2007. Clin Microbiol Infec 2011, 17, 1859-1867, doi:10.1111/j.1469-0691.2010.03456.x.

22. Chakrabarti, A.; Das, A.; Mandal, J.; Shivaprakash, M.R.; George, V.K.; Tarai, B.; Rao, P.; Panda, N.; Verma, S.C.; Sakhuja, V. The Rising Trend of Invasive Zygomycosis in Patients with Uncontrolled Diabetes Mellitus. Med Mycol 2006, 44, 335342, doi:10.1080/13693780500464930.

23. Rammaert, B.; Lanternier, F.; Zahar, J.-R.; Dannaoui, E.; Bougnoux, M.-E.; Lecuit, M.; Lortholary, O. Healthcare-Associated Mucormycosis. Clin Infect Dis Official Publ Infect Dis Soc Am 2012, 54 Suppl 1, S44-54, doi:10.1093/cid/cir867.

24. Ambrosioni, J.; Bouchuiguir-Wafa, K.; Garbino, J. Emerging Invasive Zygomycosis in a Tertiary Care Center: Epidemiology and Associated Risk Factors. Int J Infect Dis 2010, 14, e100-e103, doi:10.1016/j.ijid.2009.11.024.

25. Lanternier, F.; Dannaoui, E.; Morizot, G.; Elie, C.; Garcia-Hermoso, D.; Huerre, M.; Bitar, D.; Dromer, F.; Lortholary, O.; Group, F.M.S. A Global Analysis of Mucormycosis in France: The RetroZygo Study (2005-2007). Clin Infect Dis 2012, 54, S35-S43, doi:10.1093/cid/cir880.

26. Pagano, L.; Valentini, C.G.; Posteraro, B.; Girmenia, C.; Ossi, C.; Pan, A.; Candoni, A.; Nosari, A.; Riva, M.; Cattaneo, C.; et al. Zygomycosis in Italy: A Survey of FIMUA-ECMM (Federazione Italiana Di Micopatologia Umana Ed Animale and European Confederation of Medical Mycology). J Chemotherapy 2013, 21, 322-329, doi:10.1179/joc.2009.21.3.322. 